\title{
Improving Primary Ciliary Dyskinesia Diagnosis Using Artificial Intelligence
}

Andreia do Nascimento Pinto ${ }^{1}$, Laurens Hogeweg ${ }^{2}$, Ioannis Katramados ${ }^{2}$, Oliver Hamilton ${ }^{2}$, Amelia Shoemark ${ }^{1}$, Thomas Burgoyne ${ }^{1}$ and Claire Hogg ${ }^{1}$

${ }^{1}$ Royal Brompton Hospital, London, England, United Kingdom, ${ }^{2}$ Cosmonio, Cranfield, England, United Kingdom

\section{Introduction}

Primary ciliary dyskinesia (PCD) is an inherited autosomal-recessive disorder of motile cilia, that results in chronic lung disease, rhinosinusitis, hearing impairment and subfertility. The estimated prevalence of PCD is $\sim 1$ per 15,000 births[1], but it is more prevalent in populations where consanguinity is common. To diagnose PCD involves a combination of tests, in particular, electron microscopy (EM) that is essential for determining the type of ciliary ultrastructural defect to confirm the diagnosis. EM involves meticulous inspection of approximately 300 ciliary cross-sections, which is time-consuming and requires highly skilled and experienced diagnostic scientists. Machine learning offers an opportunity to improve diagnostic accuracy, reduce time to analyse samples, minimise the subjective element and significantly reduce costs.

Methods

NOUS, by COSMONiO, is the on-site deep-learning system that is being trained to detect the difference between healthy and abnormal cilia. Different teaching approaches are being tested that include (i) Detection of cilia and ciliary components that can be used for diagnosis (ii) Classification of normal, abnormal and useless cilia.

Results

Over 15,000 EM images of cilia have been input to Nous and the system is showing consistently improved accuracy as the training datasets enlarge. When testing it against diagnostic specialists $(n=5)$ using blinded image datasets, an agreement of $>75 \%$ in the classification of images was found. This is similar to the agreement measured between individual diagnostic specialists.

\section{Conclusions}

The training of NOUS is ongoing, and further blind datasets are being used to determine the sensitivity and specificity of this machine learning tool. Furthermore, the system is currently being trialled alongside the current diagnostic protocol, with its accuracy being accessed meticulously to validate the system for future clinical use. This tool will not only be useful for PCD diagnosis, but also in other areas of medicine.

\section{References}

[1] Amelia Shoemark, et al. Primary ciliary dyskinesia with normal ultrastructure: three-dimensional tomography detects absence of DNAH11. European Respiratory Journal 2018 51: 1701809; DOI: $10.1183 / 13993003.01809-2017$ 